\title{
A Study on the Clinical Application of Greater Omental Pedicle Flap Transplantation to Correct Anterior Resection Syndrome in Patients With Low Rectal Cancer
}

\section{haiquan qin}

Guangxi Cancer Hospital and Guangxi Medical University Affiliated Cancer Hospital https://orcid.org/0000-0001-9977-0889

\section{Linghou Meng}

Guangxi Cancer Hospital and Guangxi Medical University Affiliated Cancer Hospital

\section{Zigao Huang}

Guangxi Cancer Hospital and Guangxi Medical University Affiliated Cancer Hospital Jiankun Liao

Guangxi Cancer Hospital and Guangxi Medical University Affiliated Cancer Hospital

\section{Yan Feng}

Guangxi Cancer Hospital and Guangxi Medical University Affiliated Cancer Hospital

\section{Shanshan Luo}

Guangxi Cancer Hospital and Guangxi Medical University Affiliated Cancer Hospital

\section{Hao Lai}

Guangxi Cancer Hospital and Guangxi Medical University Affiliated Cancer Hospital

\section{Weizhong Tang}

Guangxi Cancer Hospital and Guangxi Medical University Affiliated Cancer Hospital

Xianwei Mo ( $\nabla$ mo_xianwei@126.com )

Guangxi Cancer Hospital and Guangxi Medical University Affiliated Cancer Hospital

\section{Research}

Keywords: omental pedicle flap, low anterior resection syndrome, mesorectum

Posted Date: November 20th, 2020

DOl: https://doi.org/10.21203/rs.3.rs-111298/v1

License: (c) (i) This work is licensed under a Creative Commons Attribution 4.0 International License. Read Full License 
Version of Record: A version of this preprint was published at Regenerative Therapy on December 1st, 2021. See the published version at https://doi.org/10.1016/j.reth.2021.05.003. 


\section{Abstract}

\section{Purpose}

Low anterior resection syndrome (LARS) is the most common complication after total mesorectal excision (TME) in patients with low rectal cancer and has been a challenge in colorectal surgery that severely impacts the quality of life of patients. This study aimed to introduce a revised surgical procedure which could effectively maintain rectal compliance and significantly improve LARS after the operation.

\section{Methods}

We performed mesorectal reconstruction after routine Dixon-TME using greater omental pedicle flap transplantation in 11 patients with low rectal cancer ( 5 cases of preoperative neoadjuvant chemoradiotherapy, 5 cases of preoperative neoadjuvant chemotherapy, and 1 case of postoperative adjuvant chemotherapy), thereby simulating the initial anatomical structure of the mesorectum and significantly reducing the postoperative anterior resection syndrome. The lars precision syndrome assessment scale (LARSS) was used to access the LARS.

Results

At 12 weeks after the 11 patients recovered from the anal defecation function, the average score on the LARS questionnaire was $25.5 \pm 0.5$ (mild). The average time at which anal function began to recover was $6.2 \pm 2.6$ weeks after surgery. The recovery was rapid, as the rectal and anal function of all patients generally returned to normal levels within 12 weeks, and the quality of life was close to that before surgery.

Conclusion

Greater omental flap transplantation can significantly improve LARS after Dixon-TME in patients with low rectal cancer.

\section{Introduction}

In recent years, research on low rectal cancer has progressed rapidly in many countries worldwide. With conceptual shifts in treatment, the use of laparoscopic techniques and staplers, and significant increases in rectal cancer resection, survival, and anal preservation rates in patients, Dixon-TME surgery has become the principal surgical treatment for low rectal cancer. Some patients with very low tumor position or late local staging have undergone preoperative neoadjuvant chemotherapy and other treatment methods and application of new techniques, which has increased the anal preservation rate to over 70(1). However, these patients all underwent TME, after which most experienced a series of symptoms, such as increased frequency, urgency, and difficulty in defecation, as well as incontinence; this is known as low anterior resection syndrome (2). Patients who underwent preoperative neoadjuvant chemoradiotherapy experienced LARS for longer periods of time(3), which burdened patients with physical, psychological, 
and social dysfunction and seriously affected their quality of life(4). The prevention and treatment of postoperative LARS and other complications have become a global problem. By consulting the previous literature report (5), the author found that the greater omentum has become a natural material for surgeons to repair and reconstruct the damaged tissue (6). As an ideal source of biological tissue, we cut and transplanted the greater omentum flap into the presacral space to make the omentum adhere to the presacral inflammatory site. Through postoperative MRI examination, it was found that the transplanted omentum seemed to maintain its own volume and biological characteristics in the presacral region, giving full play to the role of the circumferential mesentery of the original rectum to prevent the neorectal compliance from decreasing directly due to postoperative inflammatory adhesion between the neorectum and the presacral tissue. In this article, we reported 11 patients in which a greater omental flap was used for mesorectal reconstruction after conventional Dixon-TME to simulate the initial anatomical structure of the mesorectum. The aim of this study was to assess whether this new procedure could effectively maintain rectal compliance and significantly improve LARS after the operation.

\section{Data And Methods}

\subsection{General characteristics}

Between October 2019 and March 2020, 11 patients with low (0-7 cm from the anal margin) clinical stage II-III rectal adenocarcinoma underwent laparoscopic rectal cancer Dixon-TME surgery, after which a greater omental flap was transplanted to the presacral space to reconstruct the mesentery. All patients were pathologically confirmed and preoperatively staged by colonoscopy, computed tomography (CT), and functional magnetic resonance imaging (MRI). Preoperative neoadjuvant chemoradiotherapy was used in 4 patients with preoperative T stage $\geq 3$ and MRI indicating positive mesorectal fascia (MRF), and in 3 patients with positive $\mathrm{N}$ staging. Satisfactory reduction of the stage was achieved in all patients after neoadjuvant treatment. The basic characteristics of the patients are shown in Table 1.

\subsection{Surgical procedure}

After completing Dixon-TME with intestinal anastomosis for low rectal cancer under laparoscopic surgery, a greater omental pedicle flap was transplanted to reconstruct the mesorectum. The main technical features include the followings. Starting from the left, the greater omentum was cut along the outer gastro-omental vascular arch to the right and separated from the blood vessels at the left margin of the greater omentum. During the rightward separation, care was taken to protect the blood vessels at the right margin of the greater omentum. If the length of the omental pedicle was sufficient, the vertical blood vessels leading to the omentum from the right side of the gastro-omental vascular arch were preserved as much as possible while also separating the corresponding attachment points of the posterior lobe of the greater omentum from the corresponding location of the transverse colon. The blood vessels at the right margin of the greater omentum were used as the axis of the pedicle, and the omentum was extended downward close to the posterior peritoneum to fill the presacral space, with appropriate fixation. Care was taken to avoid the torsion of the vascular pedicle and prevent ischemia and necrosis of the greater 
omentum (Figure 1). The average time of operation for the omental flap transplantation was $15.3 \pm 1.0$ min. The amount of bleeding was not significant, and there were no complications associated with flap transplantation. Since all patients underwent an ultra-low anastomosis, a routine double-barrel ileostomy was performed after omental flap transplantation, and routine ileostomy closure was performed 8-12 weeks after surgery. 11 patients were followed up at the 12th week after ileostomy closure operation. We used the LARSS developed by Danish scholars Emmert-sen et al. in 2012 for assessing LARS(7), which was currently considered as the only tool for assessing defecation function in LARS patients in the world and validated to be an effective tool for assessing lars in clinical application in China and other countries $(8,9)$.

\section{Results}

Ten of the patients recovered uneventfully after the operation. One patient who underwent preoperative neoadjuvant chemoradiotherapy had anastomotic leakage on postoperative day 5. Digital rectal examination indicated defects in the posterior rectal wall at the rectal anastomosis for about $1 / 2$ cycle, which resolved after conservative treatment; stoma closure was performed at postoperative week 24. Imaging examinations (CT, MRI) of the pelvic floor were performed on all patients after operation, which revealed that the reconstructed mesorectum was similar in structure to the original mesorectum. The enhanced scan revealed that the greater omental blood vessels were clearly visible, indicating that the omentum is viable without liquefactive necrosis (Figures 2, 3).

After ileostomy was completed and anal defecation was recovered, the condition of LARS in the patients was dynamically recorded. It was found that the average LARS questionnaire score of the 11 patients at postoperative week 12 was $25.5 \pm 0.5$ points (mild). The average time for the recovery of conscious anal bowel control of the 11 patients was $6.2( \pm 2.6)$ weeks. The rectal and anal function of all patients generally returned to normal levels within 12 weeks, and the quality of life was close to that before the operation. One patient had anastomotic leakage but had a LARS score of 25 at 4 weeks after stoma closure, and the time for the recovery of anal bowel control was 8 weeks.

Follow-up of the 11 patients showed that the operation had significant efficacy, and rectal and anal function gradually began to recover at about postoperative week 6 , which is a significant advantage over the LARS recovery time previously reported in the literature.

\section{Discussion}

As of now, there have been few studies that reported the treatment measures which can obviously improve the LARS. It is currently believed that the mechanism of LARS is primarily related to injury to the associated muscles and nerves of the pelvic floor, changes in the volume, pressure, and compliance of the reconstructed rectum, changes in intestinal dynamics, foreign body effects, and radiotherapy. In this study, we introduced a productive way using a greater omental pedicle flap to full fill post-rectal space 
vacancy after conventional Dixon-TME, in order to improve LARS in 11 patients. The primary outcomes and feedbacks were positive and uplifting.

The main reasons for the development of LARS after anal preservation in low rectal cancer are believed to include the following. On the one hand, the large rectal ampulla is removed together with rectal cancer, and the colon intestinal segment is displaced downwards and anastomosed with the rectal stump or anal canal, thereby restoring intestinal continuity following resection of the rectum. However, the residual rectum is very short, which changes the normal physiology of the anorectum and results in the loss of the rectal anal inhibitory reflex (RAIR). In most cases, RAIR is partially restored at 12 months after the operation with the regeneration of autonomic nerves in the wall at the anastomotic scar $(10,11)$. However, the direction of peristalsis of the reconstructed rectum, the perception of feces and adjustments in distention, and the volume are very different from those of the original rectum (12). Studies have found that (13) the maximum tolerable rectal capacity after anterior rectal resection is $77 \pm 28 \mathrm{~mL}$, which is significantly less than the normal level before the operation. The decrease in the maximum tolerable volume detected by postoperative defecography and anorectal manometry of the reconstructed rectum confirmed the changes in volume and the frequency of bowel movement in the reconstructed rectum. On the other hand, the sharp separation between the presacral space and the visceral and parietal layers of the pelvic fascia during TME inevitably damages the pelvic nerve plexus branches, blood vessels, and lymphatic tissues in the mesorectum, resulting in rectal and anal dysfunction $(14,15)$. The nerve conduction function of the middle and inferior plexus of the rectum is impaired, which affects the function of the anal sphincter, resulting in a significant decrease in anal resting pressure $(16,17)$. However, we have performed preoperative CT examinations and intraoperative explorations at our center for many patients with severe long-term LARS that requires secondary surgery and found that their reconstructed rectum and presacral and pelvic organs have severe adhesions and intestinal stiffness (Figure 4). Thus, we speculate that there is no surrounding mesorectum after TME that resembles the surrounding colon that is moved down to replace the rectum, resulting in direct adhesion of the reconstructed rectum to the presacral tissue, causing the reconstructed rectum to lose peristalsis, distention, and elasticity $(18,19)$, resulting in decreased compliance and capacity tolerance in the reconstructed rectum. Over time (usually 6-12 months), the reconstructed rectum will gradually compensate by expanding, and anorectal function and compliance will improve, but not to preoperative levels $(20,21)$. A study by Bittorf et al. (22) found that rectal compliance of $1.4 \pm 1.2 \mathrm{~mL} / \mathrm{mmHg}$ at 2 years after anterior rectal resection also fully proves this point. Therefore, some researchers believe that restoring the volume and compliance of the reconstructed rectum is conducive to improving the shortterm anorectal function of patients with low anterior resection. Clinical researchers have used colon Jpouches or transverse coloplasty pouches to increase the capacity of the reconstructed rectum, but their efficacy is not significant $(23,24)$. Preoperative neoadjuvant chemoradiotherapy causes pelvic tissue fibrosis (25) and damage to the nerve plexus of the pelvic floor, which stiffens the intestinal canal, further reduces peristalsis, worsens postoperative LARS symptoms, prolongs the time for the recovery of anorectal function, and increases complications $(26,27)$. 
Therefore, we filled the presacral space with a greater omental pedicle flap to reconstruct a new mesorectum so that the reconstructed rectum has a mesenteric structure embedded in fatty tissue, which resembles the normal anatomical structure, thereby maintaining rectal compliance. Postoperative followup and evaluation using the LARS questionnaire (28) also confirmed that the operation reduced symptoms of LARS with significant efficacy, rapidly improving the symptoms to near-normal levels in a short period of time. The procedure is simple, does not significantly increase the time and difficulty of operation, has no significant complications, and is suitable for broad application. In one patient, due to poor healing of the anastomosis (nearly $1 / 2$ cycle) in the intestinal canal after the operation, the transplanted omentum may experience limited inflammation, thereby promoting healing. However, due to the small number of cases, long-term observation is still necessary. Even more importantly, the patient's anal function quickly returned to normal after the operation, whereas low rectal cancer patients with anastomotic leakage in the past have experienced a series of symptoms such as increased defecation frequency, urgency, difficulty in defecation, and incontinence due to pelvic inflammation, from which recovery is difficult. Other studies have found that the particular biological characteristics of the greater omentum can be used to treat brain injuries, hand trauma, and other defects, and has the ability to repair nerve tissue and promote regeneration $(6,29,30)$. Research on whether the omental pedicle flap reconstruction of the mesorectum in the present study has similar restoration of neurological function as to accelerate the recovery of anal function is still lacking.

Greater omental pedicle flap transplantation for filling the presacral space and reconstructing the mesorectum can significantly improve LARS after Dixon-TME in patients with low rectal cancer. The flap comes from the body itself and the material is natural. The surgical procedure is simple and does not significantly increase the time and difficulty of operation. There are no significant complications associated with omental transplantation. Therefore, we believe that this is a promising technique warranting application. However, the number of cases treated with this technique is still small. After further studies with larger sample sizes, we expect it to become a standard surgical procedure for prevention of LARS after Dixon-TME for low rectal cancer, drive the development of functional organ surgery, and have a positive impact on tissue and organ function restoration and other complex problems.

\section{Conclusion}

The greater omentum flap transplantation can significantly improve LARS after Dixon-TME in patients with low rectal cancer, which may become the standard surgical procedure for the prevention of LARS after rectal cancer surgery. A larger sample size is needed to further verify its safety and effectiveness.

\section{Abbreviations}

LARS: Low anterior resection syndrome, TME: Total mesorectal excision; LARSS: Lars precision syndrome assessment scale, CT: Computed tomography, MRI: magnetic resonance imaging, MRF: mesorectal fascia, RAIR: rectal anal inhibitory reflex. 


\section{Declarations}

\section{Ethics approval}

The ethics review committee of Guangxi Medical University Cancer Hospital approved the present study.

\section{Consent for publication}

Written informed consent for this research was obtained from the patient prior to surgery. The patient has provided written permission for the publication.

\section{Availability of data and materials}

The data used and/or analyzed in this study are available from the corresponding author on reasonable request.

\section{Competing interests}

The authors declare that they have no competing interests.

\section{Funding}

This study was funded by the Self-Financing Research Project of the Health and Family Planning Commission of Guangxi Zhuang Autonomous Region (Z2015607); the Guangxi Medical and Health Appropriate Technology Development and Promotion Application Project (S2017098); 2019 Guangxi University High-level Innovation Team and the Project of Outstanding Scholars Program, and Guangxi Science and Technology Project (2019AC03004); Guangxi Science and Technology Base and Talent Project (AD19245197).

\section{Authors' contributions}

All authors contributed to the study conception and design. HQ, LM and XM conceived the study. Secondly, ZH, JL, SL and HL contributed to data acquisition and analysis. HQ, LM and YF interpreted data and drafted the manuscript. Finally, XM and WT revised the the manuscript and proved the final version. And all of the authors revised and approved the manuscirpt.

\section{Acknowledgements}

I'd like to express my sincere thanks to all those who have lent me hands in the course of my writing this paper囚as well as Editage (www.editage.cn) for their work in English language editing.

\section{References}

1. Miller KD, Siegel RL, Lin CC, Mariotto AB, Kramer JL, Rowland JH, et al. Cancer treatment and survivorship statistics, 2016. CA: a cancer journal for clinicians. 2016;66(4):271-89. 
2. Bryant CL, Lunniss PJ, Knowles CH, Thaha MA, Chan CL. Anterior resection syndrome. The Lancet Oncology. 2012;13(9):e403-8.

3. Croese AD, Zubair ON, Lonie J, Trollope AF, Vangaveti VN, Mushaya C, et al. Prevalence of low anterior resection syndrome at a regional Australian centre. ANZ J Surg. 2018;88(12):E813-e7.

4. Viola KV, Ariyan C, Sosa JA. Surgical perspectives in gastrointestinal disease: a study of quality of life outcomes in esophageal, pancreatic, colon, and rectal cancers. World J Gastroenterol. 2006;12(20):3213-8.

5. Desnoo L, Faithfull S. A qualitative study of anterior resection syndrome: the experiences of cancer survivors who have undergone resection surgery. Eur J Cancer Care (Engl). 2006;15(3):244-51.

6. Di Nicola V. Omentum a powerful biological source in regenerative surgery. Regenerative therapy. 2019;11:182-91.

7. Emmertsen KJ, Laurberg S. Low anterior resection syndrome score: development and validation of a symptom-based scoring system for bowel dysfunction after low anterior resection for rectal cancer. Ann Surg. 2012;255(5):922-8.

8. Hou XT, Pang D, Lu Q, Yang P, Jin SL, Zhou YJ, et al. Validation of the Chinese version of the low anterior resection syndrome score for measuring bowel dysfunction after sphincter-preserving surgery among rectal cancer patients. Eur J Oncol Nurs. 2015;19(5):495-501.

9. Therese J. International validation of the low anterior resection syndrome score. Annals of surgery. 2014;4(259).

10. O'Riordain MG, Molloy RG, Gillen P, Horgan A, Kirwan WO. Rectoanal inhibitory reflex following low stapled anterior resection of the rectum. Dis Colon Rectum. 1992;35(9):874-8.

11. Horgan AF, Molloy RG, Coulter J, Sheehan M, Kirwan WO. Nerve regeneration across colorectal anastomoses after low anterior resection in a canine model. Int J Colorectal Dis. 1993;8(3):167-9.

12. van Duijvendijk P, Slors F, Taat CW, Heisterkamp SH, Obertop H, Boeckxstaens GE. A prospective evaluation of anorectal function after total mesorectal excision in patients with a rectal carcinoma. Surgery. 2003;133(1):56-65.

13. Morihiro M, Koda K, Seike K, Miyauchi H, Miyazaki M. Characteristic findings on defecography according to reconstruction method and defecatory disorder following sphincter-saving surgery for rectal cancer. Int J Colorectal Dis. 2008;23(9):883-92.

14. Tuson JR, Everett WG. A retrospective study of colostomies, leaks and strictures after colorectal anastomosis. Int J Colorectal Dis. 1990;5(1):44-8.

15. Pucciani F. A review on functional results of sphincter-saving surgery for rectal cancer: the anterior resection syndrome. Updates Surg. 2013;65(4):257-63.

16. Pucciani F, Bologna A, Cianchi F, Cortesini C. Anorectal Physiology following Sphincter-Saving Operations for Rectal Cancer. Digestive Surgery. 1993;10(1):33-8.

17. Rocca Rossetti S. Functional anatomy of pelvic floor. Archivio italiano di urologia, andrologia : organo ufficiale [di] Societa italiana di ecografia urologica e nefrologica. 2016;88(1):28-37. 
18. Romaniszyn M, Richter P, Walega P, Kenig J, Nowak M, Nowak W. Low-anterior-resection syndrome. How does neoadjuvant radiotherapy and low resection of the rectum influence the function of anal sphincters in patients with rectal cancer? Preliminary results of a functional assessment study. Pol Przegl Chir. 2012;84(4):177-83.

19. lizuka I, Koda K, Seike K, Shimizu K, Takami Y, Fukuda H, et al. Defecatory malfunction caused by motility disorder of the neorectum after anterior resection for rectal cancer. Am J Surg. 2004;188(2):176-80.

20. Nesbakken A, Nygaard K, Lunde OC. Mesorectal excision for rectal cancer: functional outcome after low anterior resection and colorectal anastomosis without a reservoir. Colorectal Dis. 2002;4(3):1726.

21. Lee SJ, Park YS. Serial evaluation of anorectal function following low anterior resection of the rectum. Int J Colorectal Dis. 1998;13(5-6):241-6.

22. Bittorf B, Stadelmaier U, Göhl J, Hohenberger W, Matzel KE. Functional outcome after intersphincteric resection of the rectum with coloanal anastomosis in low rectal cancer. Eur $\mathrm{J}$ Surg Oncol. 2004;30(3):260-5.

23. Ulrich A, Z'Graggen K, Weitz J, Büchler MW. Functional results of the colon J-pouch versus transverse coloplasty pouch in Heidelberg. Recent results in cancer research Fortschritte der Krebsforschung Progres dans les recherches sur le cancer. 2005;165:205-11.

24. Rink AD, Sgourakis G, Sotiropoulos GC, Lang H, Vestweber KH. The colon J-pouch as a cause of evacuation disorders after rectal resection: myth or fact? Langenbecks Arch Surg. 2009;394(1):79-91.

25. Canda AE, Terzi C, Gorken IB, Oztop I, Sokmen S, Fuzun M. Effects of preoperative chemoradiotherapy on anal sphincter functions and quality of life in rectal cancer patients. Int $\mathrm{J}$ Colorectal Dis. 2010;25(2):197-204.

26. Pollack J, Holm T, Cedermark B, Holmström B, Mellgren A. Long-term effect of preoperative radiation therapy on anorectal function. Dis Colon Rectum. 2006;49(3):345-52.

27. Ammann K, Kirchmayr W, Klaus A, Mühlmann G, Kafka R, Oberwalder M, et al. Impact of neoadjuvant chemoradiation on anal sphincter function in patients with carcinoma of the midrectum and low rectum. Archives of surgery (Chicago, III : 1960). 2003;138(3):257-61.

28. Shah S, Lowery E, Braun RK, Martin A, Huang N, Medina M, et al. Cellular basis of tissue regeneration by omentum. PLoS One. 2012;7(6):e38368.

29. Goldsmith HS, Chen WF, Duckett SW. Brain vascularization by intact omentum. Archives of surgery (Chicago, III : 1960). 1973;106(5):695-8.

30. Żyluk A, Szlosser Z, Puchalski P. The results of the treatment of hand - Outcomes of the treatment of hand degloving injuries with greater omentum flaps. Pol Przegl Chir. 2019;91(6):20-7.

\section{Tables}

Table 1. Baseline characteristics of 11 patients with low rectal adenocarcinoma 


\begin{tabular}{|c|c|}
\hline Parameters & $\mathrm{n}(\%)$ \\
\hline \multicolumn{2}{|l|}{ Sex } \\
\hline Male & $8(72.72)$ \\
\hline Female & $3(27.27)$ \\
\hline Age, median (range) & $50(32-74)$ \\
\hline BMI $\left(\mathrm{kg} / \mathrm{m}^{2}\right)$, median (range) & $18.9(15.5-22)$ \\
\hline \multicolumn{2}{|l|}{ ASA } \\
\hline ASA score 1 & $7(63.63)$ \\
\hline ASA score 2 & $4(36.36)$ \\
\hline Distance to anal Verge (mm), median (range) & $48(20-70)$ \\
\hline Tumor size (mm), media (range) & $31(17-48)$ \\
\hline \multicolumn{2}{|l|}{ AJCC clinical Stages } \\
\hline Stage $\otimes$ & 0 \\
\hline 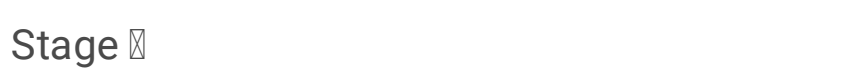 & $5(45.45)$ \\
\hline Stage $\otimes$ & $6(54.54)$ \\
\hline 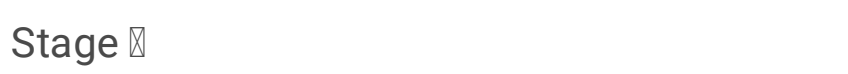 & 0 \\
\hline \multicolumn{2}{|l|}{ Clinical N stage } \\
\hline cNO & $6(54.54)$ \\
\hline cN1 & $3(18.18)$ \\
\hline cN2 & $2(27.27)$ \\
\hline \multicolumn{2}{|l|}{ Neoadjuvant treatment } \\
\hline Chemotherapy & $5(45.45)$ \\
\hline
\end{tabular}

Page 11/19 


\begin{tabular}{|ll|} 
Radiation therapy & 0 \\
\hline Radiation and chemotherapy & $5(45.45)$ \\
\hline
\end{tabular}

ASA: American Society of Anesthesiologists, BMI: Body mass index, AJCC: The American Joint Committee on Cancer.

\section{Figures}
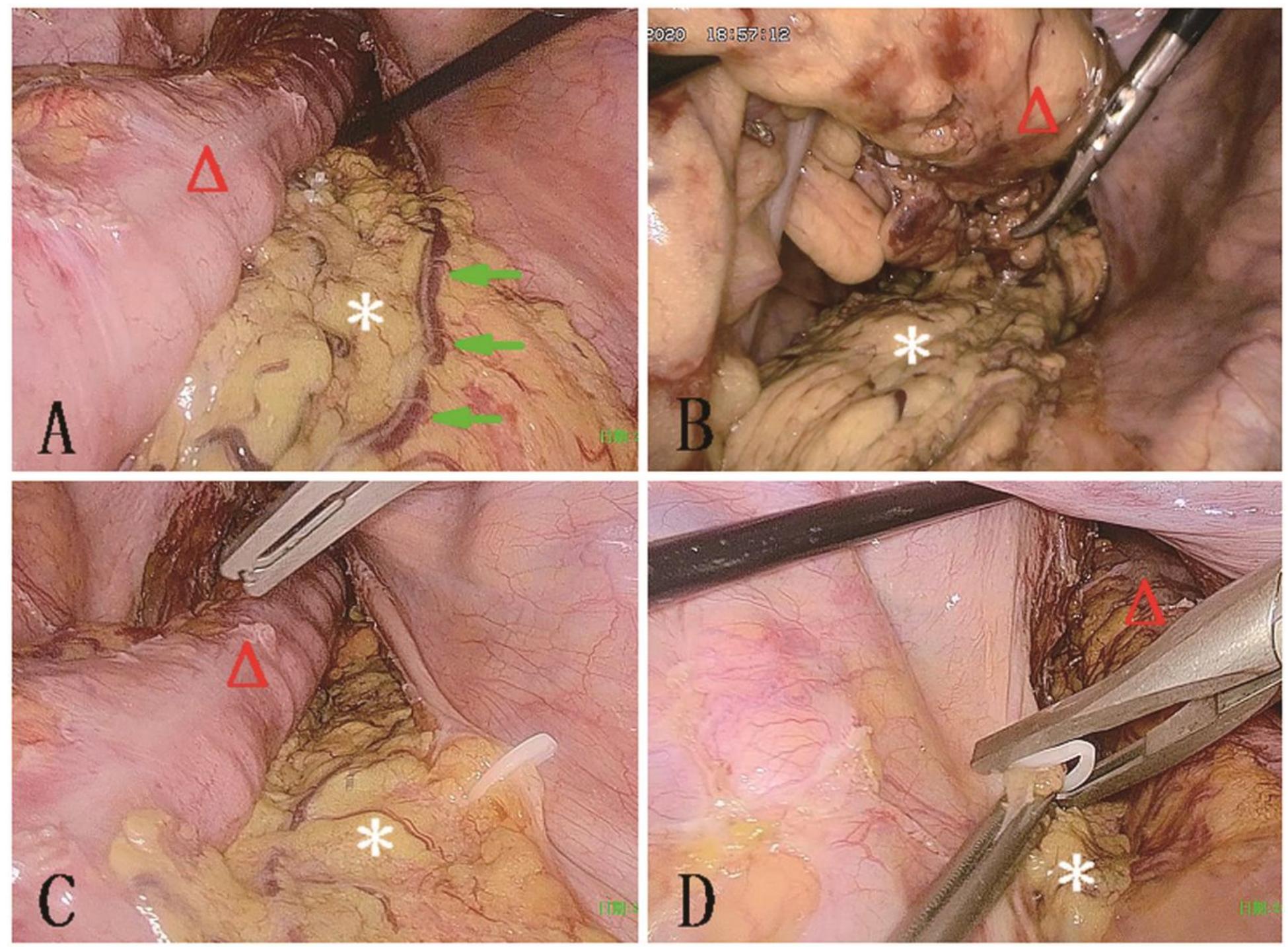

\section{Figure 1}

The main steps of the operation. a) Greater omental pedicle transplantation in the pelvic cavity. b) Greater omentum padding behind the "new rectum." c) Tissue clips are used to fix the greater omentum to the peritoneum of the right pelvic wall. d) Tissue clips are used to fix the greater omentum to the peritoneum of the left pelvic wall. Image labels: ${ }^{*}$, greater omentum with the vascular pedicle. $\Delta$, the intestinal canal of the "new rectum". Green arrows, omental blood vessels. 

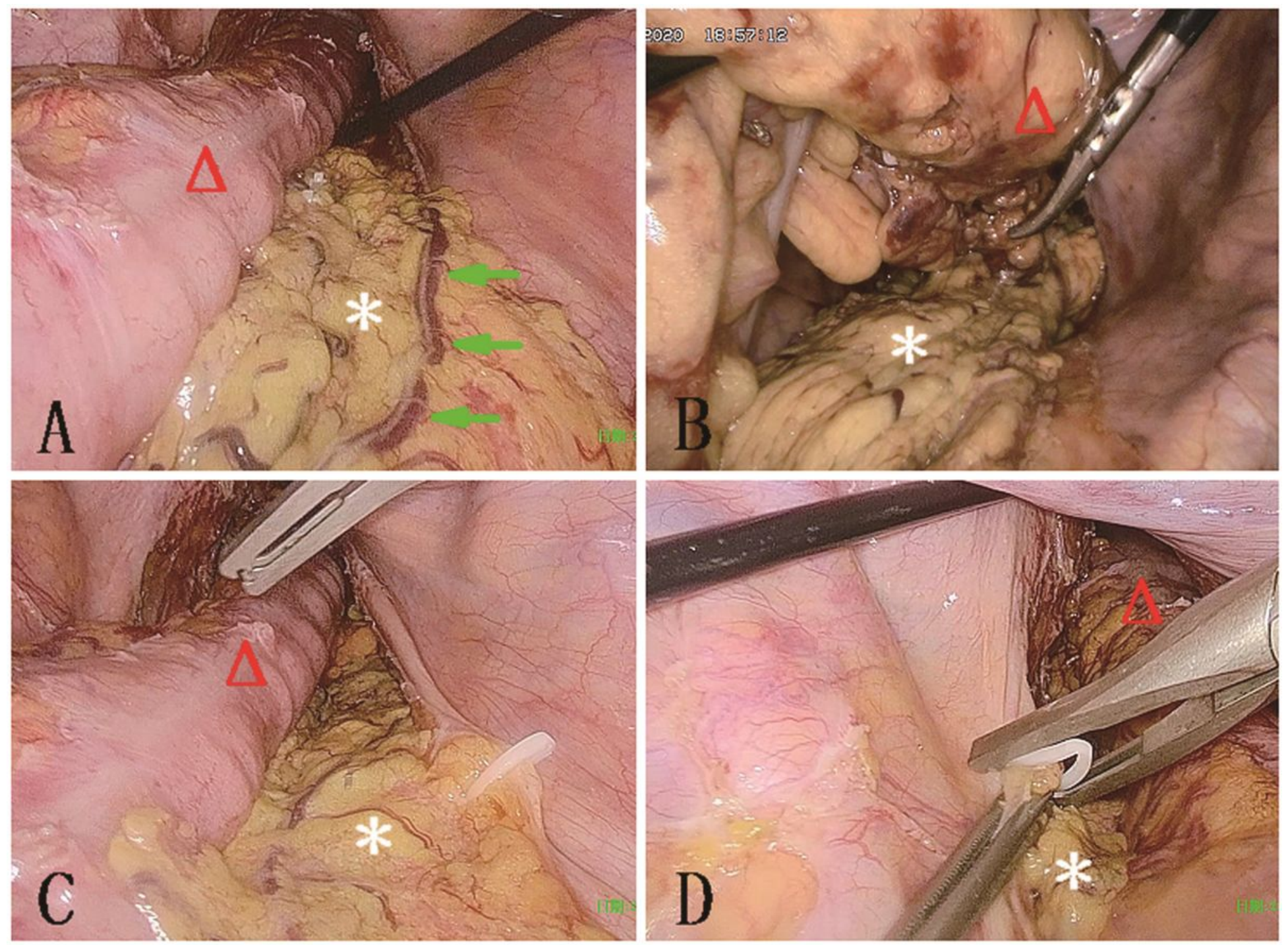

\section{Figure 1}

The main steps of the operation. a) Greater omental pedicle transplantation in the pelvic cavity. b) Greater omentum padding behind the "new rectum." c) Tissue clips are used to fix the greater omentum to the peritoneum of the right pelvic wall. d) Tissue clips are used to fix the greater omentum to the peritoneum of the left pelvic wall. Image labels: *, greater omentum with the vascular pedicle. $\Delta$, the intestinal canal of the "new rectum". Green arrows, omental blood vessels. 

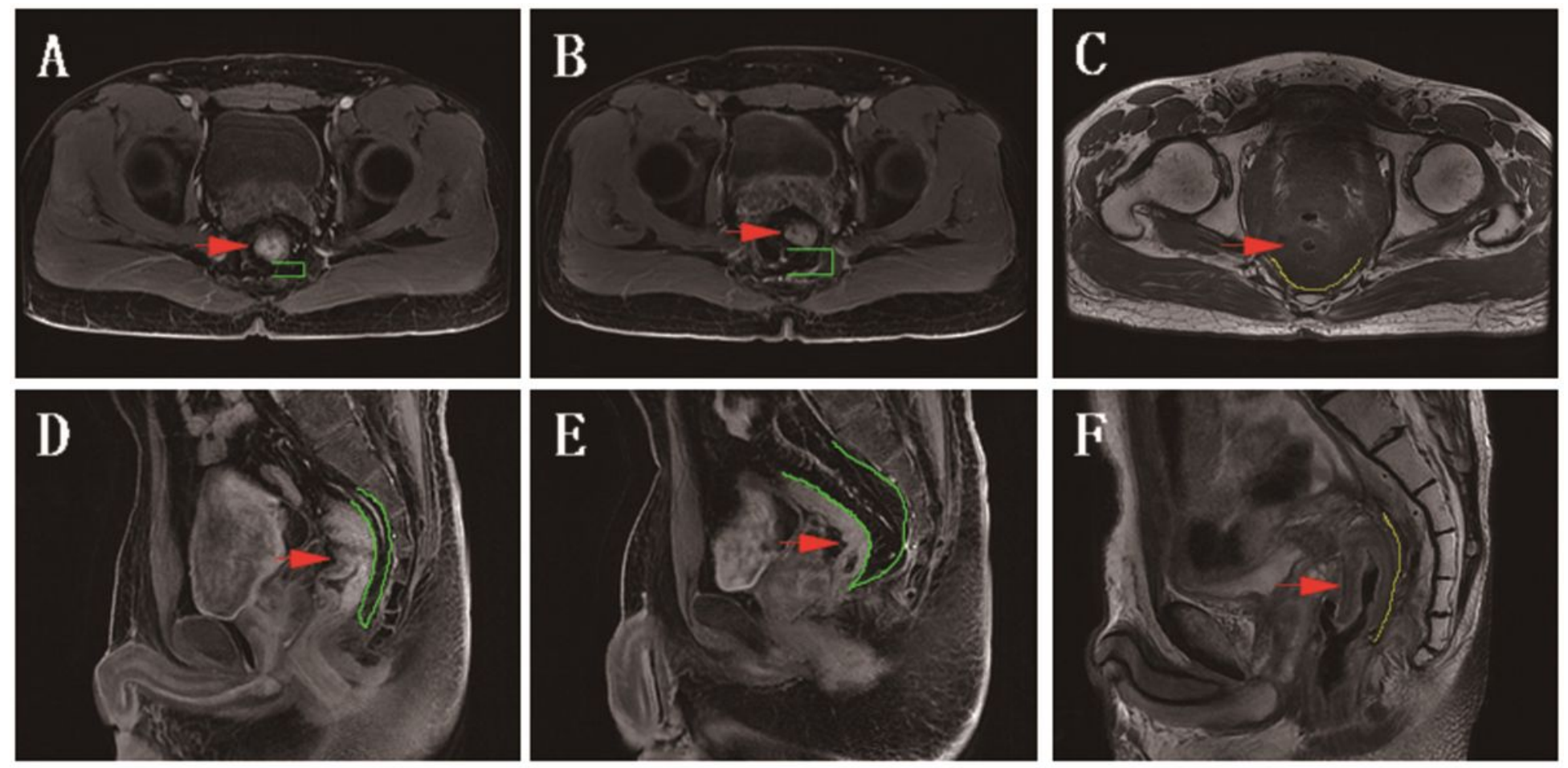

Figure 2

Rectal MRI examination shows posterior mesorectum before/after the operation and post-operation control image. a, d) posterior mesorectum before the operation. b, e) Greater omental padding behind the new rectum after greater omental pedicle flap transplantation, shows ideal posterior rectal space-filling efficacy. $c, f)$ No flap after radiotherapy, shows direct adhesion of the new rectum to the anterior sacrum. Image labels: red arrow, a/d: rectal cancer; b/e, c/f: new rectum. Green $\Pi$ / green outline, the thickness of the posterior mesorectum or greater omentum behind the new rectum. Yellow outline, direct adhesion of the new rectum line. 

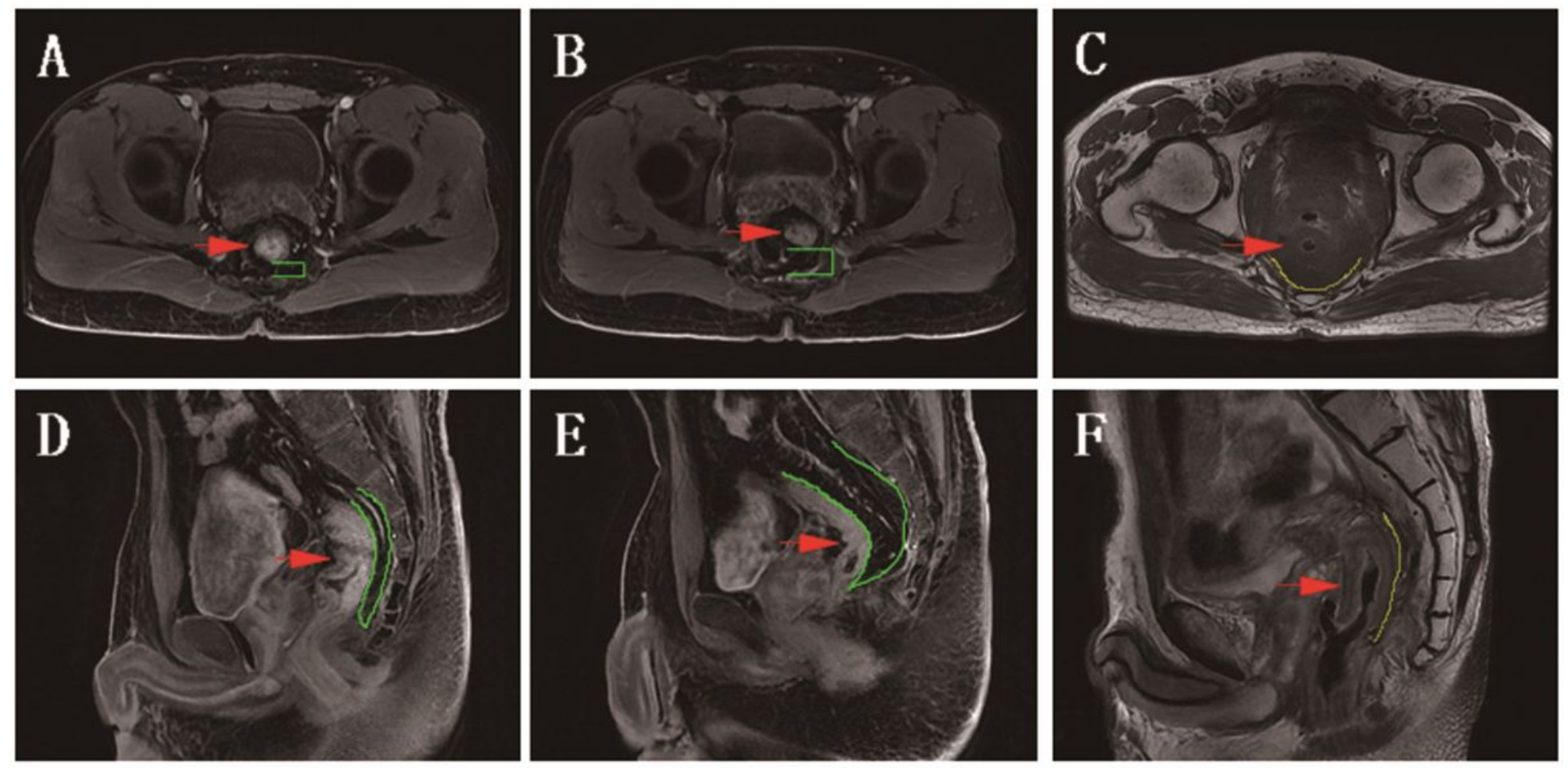

Figure 2

Rectal MRI examination shows posterior mesorectum before/after the operation and post-operation control image. a, d) posterior mesorectum before the operation. b, e) Greater omental padding behind the new rectum after greater omental pedicle flap transplantation, shows ideal posterior rectal space-filling efficacy. $c, f)$ No flap after radiotherapy, shows direct adhesion of the new rectum to the anterior sacrum. Image labels: red arrow, a/d: rectal cancer; b/e, c/f: new rectum. Green $\Pi$ / green outline, the thickness of the posterior mesorectum or greater omentum behind the new rectum. Yellow outline, direct adhesion of the new rectum line. 

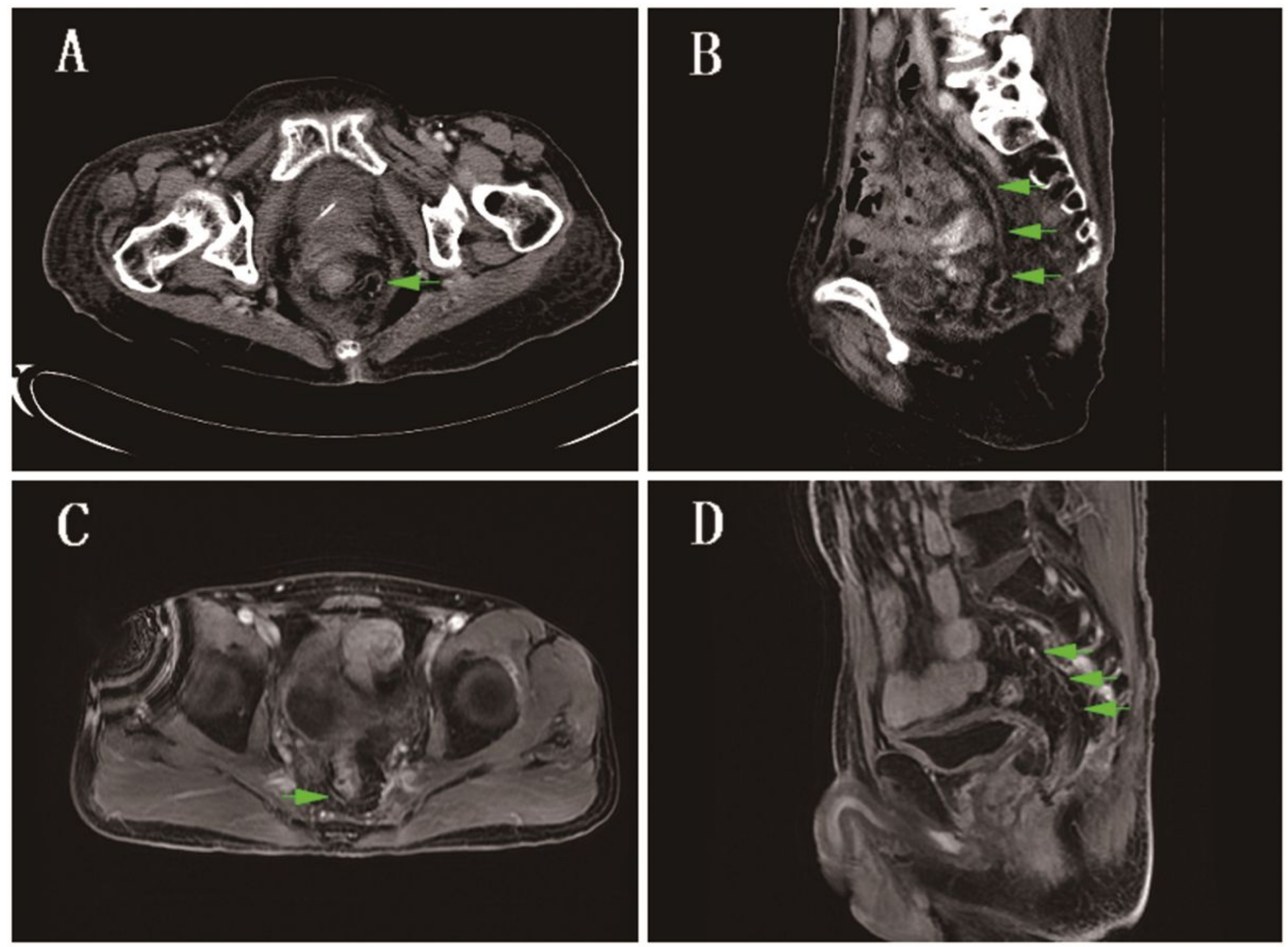

\section{Figure 3}

Enhanced computed tomography (CT) of the pelvic cavity and rectal magnetic resonance imaging (MRI) show that the flaps have a good blood supply after greater omental pedicle flap transplantation and ideal posterior rectal space-filling efficacy. Image labels: green arrow, omental blood vessels. 

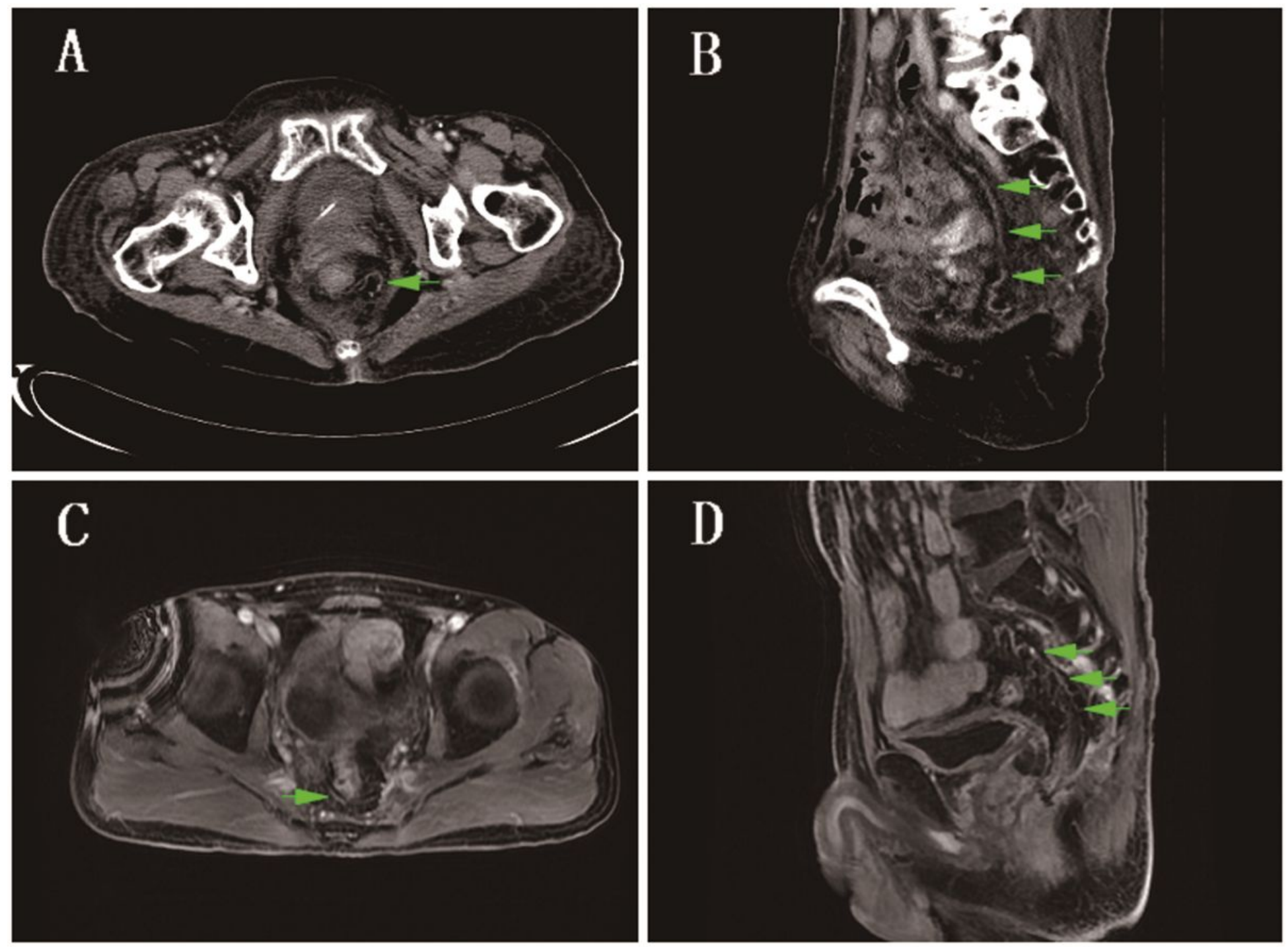

\section{Figure 3}

Enhanced computed tomography (CT) of the pelvic cavity and rectal magnetic resonance imaging (MRI) show that the flaps have a good blood supply after greater omental pedicle flap transplantation and ideal posterior rectal space-filling efficacy. Image labels: green arrow, omental blood vessels. 


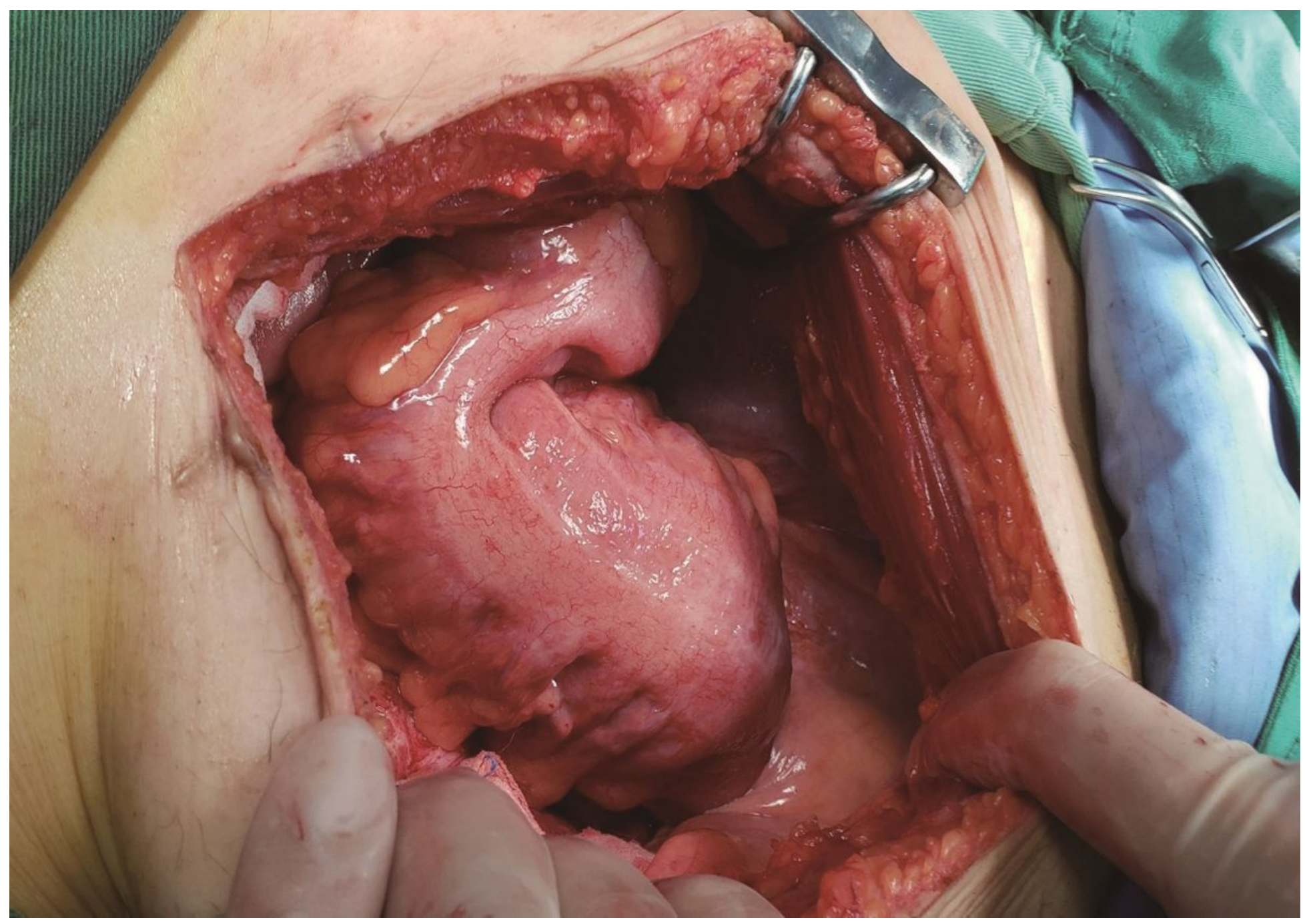

Figure 4

A second open-abdomen operation reveals severe adhesion between the new rectum and the pelvic floor in the abdominal cavity. 


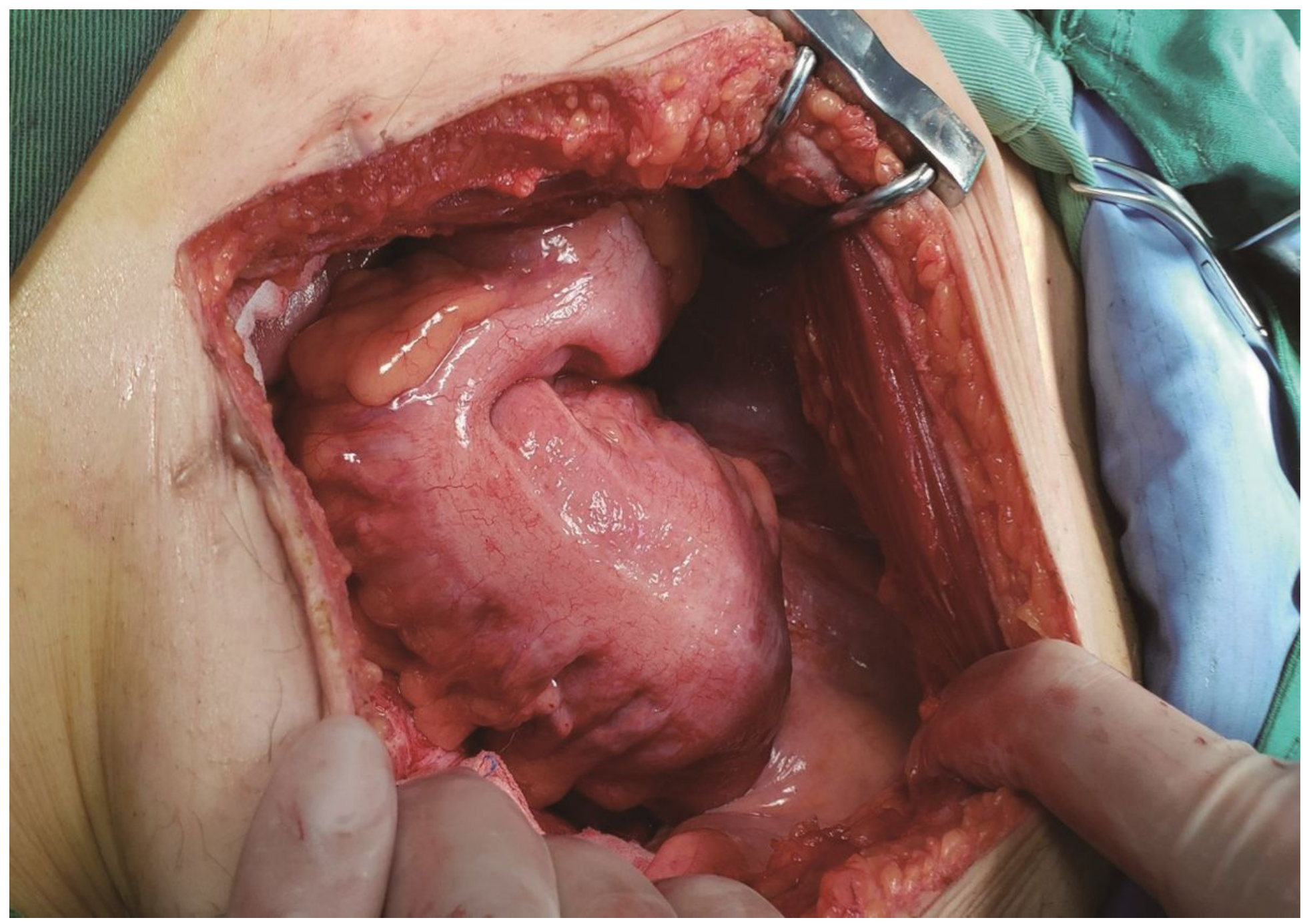

Figure 4

A second open-abdomen operation reveals severe adhesion between the new rectum and the pelvic floor in the abdominal cavity. 